\title{
STonKGs: a sophisticated transformer trained on biomedical text and knowledge graphs
}

\author{
Helena Balabin $\mathbb{D}^{1,2, *}$, Charles Tapley Hoyt ${ }^{3}{ }^{3}$, Colin Birkenbihl ${ }^{1}{ }^{1}$, \\ Benjamin M. Gyori $\mathbb{D}^{3}{ }^{3}$, John Bachman $\mathbb{D}^{3}{ }^{3}$, Alpha Tom Kodamullil $\mathbb{D}^{1}$, \\ Paul G. Plöger $\mathbb{D}^{2}$, Martin Hofmann-Apitius $\mathbb{D}^{1}$ and Daniel Domingo-Fernández

\begin{abstract}
${ }^{1}$ Department of Bioinformatics, Fraunhofer Institute for Algorithms and Scientific Computing, 53757 Sankt Augustin, Germany, ${ }^{2}$ Department of Bonn-Rhein-Sieg, University of Applied Sciences, 53757 Sankt Augustin, Germany, ${ }^{3}$ Laboratory of Systems Pharmacology, Harvard Medical School, Boston, MA 02115, USA, ${ }^{4}$ Fraunhofer Center for Machine Learning, Sankt Augustin, Germany and ${ }^{5}$ Enveda Biosciences, Boulder, CO 80301, USA
\end{abstract}

*To whom correspondence should be addressed.

Associate Editor: Jonathan Wren

Received on August 18, 2021; revised on December 9, 2021; editorial decision on December 26, 2021; accepted on January 3, 2022

\begin{abstract}
Motivation: The majority of biomedical knowledge is stored in structured databases or as unstructured text in scientific publications. This vast amount of information has led to numerous machine learning-based biological applications using either text through natural language processing (NLP) or structured data through knowledge graph embedding models. However, representations based on a single modality are inherently limited.

Results: To generate better representations of biological knowledge, we propose STonKGs, a Sophisticated Transformer trained on biomedical text and Knowledge Graphs (KGs). This multimodal Transformer uses combined input sequences of structured information from KGs and unstructured text data from biomedical literature to learn joint representations in a shared embedding space. First, we pre-trained STonKGs on a knowledge base assembled by the Integrated Network and Dynamical Reasoning Assembler consisting of millions of text-triple pairs extracted from biomedical literature by multiple NLP systems. Then, we benchmarked STonKGs against three baseline models trained on either one of the modalities (i.e. text or KG) across eight different classification tasks, each corresponding to a different biological application. Our results demonstrate that STonKGs outperforms both baselines, especially on the more challenging tasks with respect to the number of classes, improving upon the F1-score of the best baseline by up to 0.084 (i.e. from 0.881 to 0.965 ). Finally, our pre-trained model as well as the model architecture can be adapted to various other transfer learning applications.

Availability and implementation: We make the source code and the Python package of STonKGs available at GitHub (https://github.com/stonkgs/stonkgs) and PyPI (https://pypi.org/project/stonkgs/). The pre-trained STonKGs models and the task-specific classification models are respectively available at https://huggingface.co/stonkgs/ stonkgs-150k and https://zenodo.org/communities/stonkgs.
\end{abstract}

Contact: helena.balabin@gmail.com or daniel.domingo.fernandez@scai.fraunhofer.de

Supplementary information: Supplementary data are available at Bioinformatics online.

\section{Introduction}

In recent years, the availability of biomedical data has increased drastically (Dash et al., 2019). Such data originate from a vast collection of modalities such as high-throughput experiments, clinical text documents as well as cell-based and biochemical assay data. The information derived from research carried out on those data is commonly stored in two distinct forms: (i) as unstructured free text in scientific publications, and (ii) in condensed, structured biomedical networks. However, the biology represented in the literature strongly depends on the different contexts that it occurs in. For instance, certain proteins or chemicals may exclusively interact with others in a specific tissue or cell type (Stacey et al., 2018), or specific biochemical reactions may only take place under certain conditions. Consequently, to exploit the biomedical knowledge stored in both structured and unstructured formats, it is crucial to study each relation in the relevant context it was observed in. While networks often lack this contextual 
information due to their inherent degree of abstraction (Saqi et al., 2019), unstructured text contains context at the expense of explicit logical structure. Thus, the complementary strengths from both sources could be leveraged to enable a more complete, context-specific and actionable representation of biological knowledge.

Biomedical knowledge graphs (KGs) represent information in a structured manner to encode the broad spectrum of complex interactions occurring in biology. To exploit the information contained in KGs through machine learning algorithms, numerous knowledge graph embedding models (KGEMs) have been developed to encode the entities and relations of KGs in a higher-dimensional vector space while attempting to retain their structural properties (Ji et al., 2021). Utilizing the resulting vector representations, more sophisticated tasks can be conducted (i.e. link prediction, node classification and graph classification). When these KGs contain more detailed, contextualized descriptions of biological interactions (e.g. the type of interaction and the conditions in which it occurs), the performance of KGEMs can be substantially improved. Such improvements can be achieved by incorporating metadata that specifies the context of each relation (e.g. the $\mathrm{pH}$ value in which a molecular interaction occurs or the specific cell type in which a protein is expressed). Therefore, context-specific KGs have recently been used in combination with other data modalities in several biomedical applications. For instance, Federico and Monti (2021) demonstrated how to gain insights on specific human cell-line processes by annotating proteinprotein interaction networks with contextualized cell-line information extracted from the scientific literature. Similarly, a recent study from Doncheva et al. (2021) introduced a methodology that proposes the most suitable organism to model a human pathway by evaluating whether the expression of genes in a certain pathway across four species (i.e. rat, mouse, pig and humans) is maintained in the same tissue. To achieve this, the authors leveraged a contextualized protein-protein interaction network generated with ortholog information together with transcriptomics data and mentions of proteins in the scientific publications.

Due to the availability and abundance of unstructured text data in scientific literature, natural language processing (NLP) has become an important tool for extracting information on biomedical contexts. Similar to KGEMs, language models (LMs) are used to transform their input, namely word sequences, into a highdimensional vector space, resulting in so-called embeddings. One approach to learning these embeddings in a contextualized manner is through the use of the attention mechanism (Vaswani et al., 2017), which is, for instance, used in the Bidirectional Encoder Representations from Transformers (BERT) model by Devlin et al. (2019). Its biomedical counterpart, BioBERT (Lee et al., 2019), is pre-trained on a large PubMed text corpus to learn a contextualized representation of biomedical knowledge. Such a pre-trained Transformer can then be used on a variety of classification tasks (e.g. named entity recognition (Li et al., 2016), sequence classification (Baker et al., 2016) and question answering (Tsatsaronis et al., 2015)) with minimal model architecture adaptations in a so-called fine-tuning procedure. The goal is to leverage and flexibly adapt the pre-trained embedding representations, which is especially beneficial for fine-tuning tasks with small training datasets. For example, multiple pre-trained Transformer-based models achieved state-of-the-art performances on tasks with small datasets encompassed in the General Language Understanding and Evaluation (GLUE) benchmark (Wang et al., 2018).

To incorporate other data modalities, Transformers with crossmodal attention have been proposed as an extension to purely textbased Transformer models. For instance, Tsai et al. (2019) used cross-modal attention to capture complex interdependencies between text, video and audio data to enhance the frame of reference of context-specific LMs. More recently, Kamath et al. (2021) improved state-of-the-art performances on multiple visual reasoning tasks by applying a cross encoder on a concatenation of textual and visual embeddings. Moreover, several Transformer-based LMs have demonstrated the benefit of incorporating structured KG data in the general (Zhang et al., 2019) as well as the biomedical domain (Fei et al., 2020; He et al., 2020).In addition, there are several approaches for combining KG and text data that are not based on Transformers (e.g. Toutanova et al., 2015; Wang et al., 2014). However, the former approaches operate at a word level (rather than sentence level) by combining textual embeddings from LMs and entity embeddings from KGs through entity linking (i.e. the process of aligning text tokens and KG entities). Recently, Sun et al. (2020) proposed a different strategy for combining information from KGs and text by concatenating word, entity and relation embeddings at the sentence level. Similarly, Nadkarni et al. (2021) have combined textual descriptions of nodes with embedding representations learned by KGEMs for link prediction. Finally, Transformer-based LMs have also been directly applied on graphstructured data (Ying et al., 2021).

Here, we present STonKGs, a Sophisticated Transformer trained on biomedical text and KGs. STonKGs is a multimodal approach that combines subgraph-level information from a KG with corresponding sentence-level text data from the literature, improving upon previous embedding representations by encompassing the two complementary data sources in a shared embedding space. We demonstrate STonKGs on a KG consisting of millions of text-triple pairs extracted from the biomedical literature and pathway databases, assembled using the Integrated Network and Dynamical Reasoning Assembler (INDRA) (Gyori et al., 2017). Using this dataset, we benchmark STonKGs against two baseline models [i.e. BioBERT (Lee et al., 2019) and node2vec (Grover and Leskovec, 2016)] in a transfer learning setting on eight different fine-tuning tasks corresponding to distinct biological applications. Our results highlight how combining both modalities can enable STonKGs to outperform both baselines, particularly the more complex classification tasks (i.e. those with the larger number of classes). Furthermore, the STonKGs model architecture can be easily adapted to other applications on text-triple pairs in the biomedical as well as other distinct domains. We released the source code and pre-trained STonKGs models at https://github.com/stonkgs/ stonkgs and https://hugging face.co/stonkgs/stonkgs-150k.

\section{Materials and methods}

Our main goal was to evaluate the effect of combining text and KG data in the proposed model architecture (i.e. STonKGs). As a data resource, we used the INDRA KG, which contains millions of triples with text evidence and annotations, further described in Section 2.1 (Fig. 1A). We compared our proposed STonKGs model against three baseline models which only used one of the respective knowledge sources in a unified experimental setting (see Section 2.2 and Fig. 1B). Next, we outline our evaluation setting consisting of eight different classification tasks (Section 2.3). Finally, we describe the software implementation and hardware used to conduct this work in Section 2.4.

\subsection{Dataset}

To combine the structured information represented in a KG with unstructured text, we required a KG containing relations for each triple and the corresponding text evidence from which the triple has been extracted. As a result, our dataset consisted of text-triple pairs such as ('Sorafenib is a multi-kinase inhibitor that inhibits various kinases including VEGFR-2', (Sorafenib, directlyDecreases, VEGFR-2), which is represented as (a(pubchem.compound : 216239), directly Decreases, $\operatorname{kin}(\mathrm{p}($ hgnc : 6307)) )). We used a KG containing 35150093 triples assembled by INDRA (Gyori et al., 2017) from pathway databases and the output of text mining systems (Supplementary Table S1) run on (i) PubMed abstracts, (ii) PubMed Central full text articles and (iii) several publishers' text mining corpora available as of April 2021 (see Supplementary Fig. S1 for details on node and relation types). The original version of the INDRA KG comprised non-grounded nodes (i.e. nodes that could not be normalized to a standardized ontology) and triples without text evidence, both of which were filtered out in a preliminary data cleaning step (described in Supplementary Text S1). Ultimately, the 


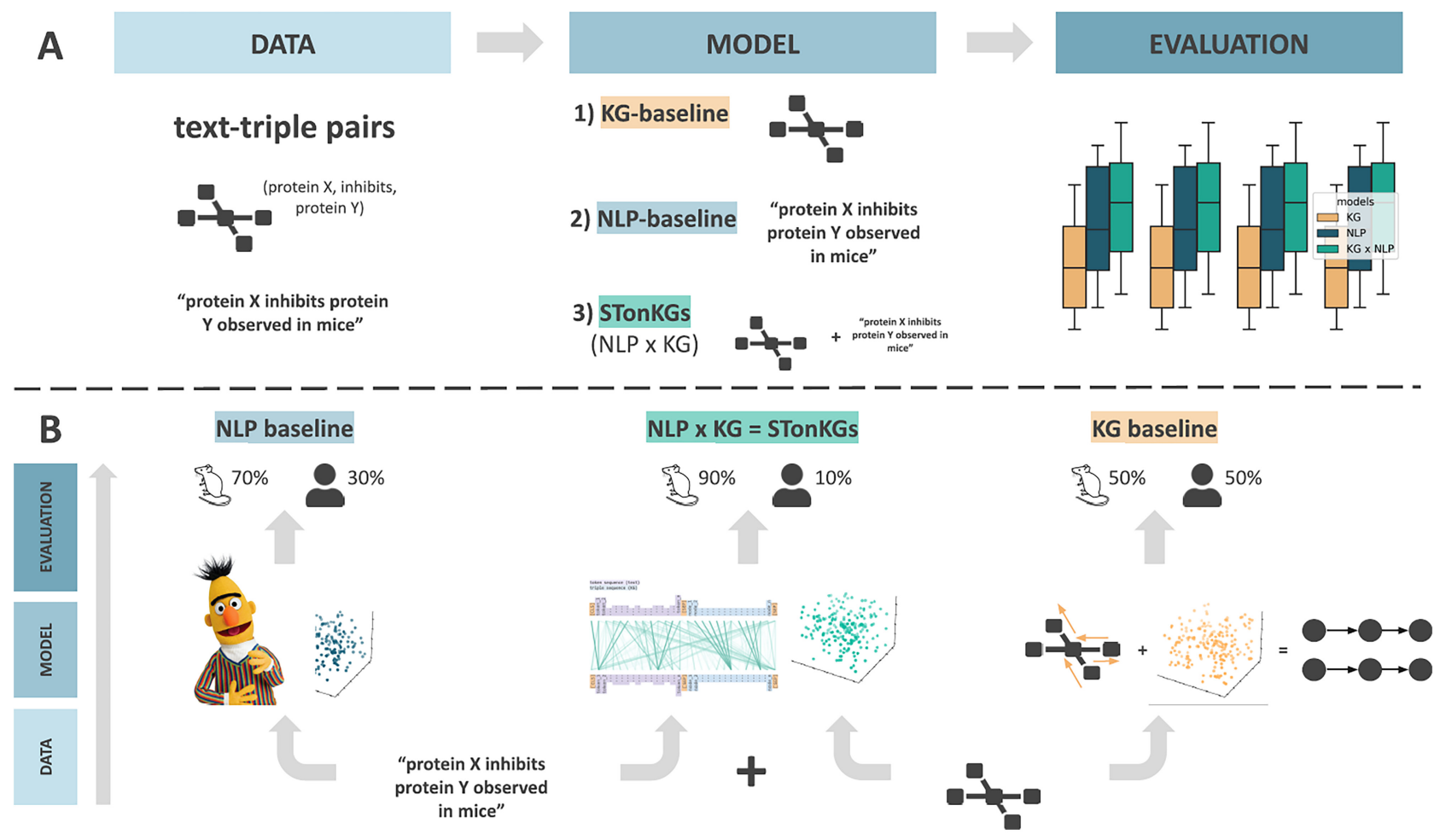

Fig. 1. Methodology workflow. This figure illustrates the classification of the context annotation for a given text-triple pair. In this example, the models aim to predict the species in which a certain biological process was observed (e.g. mice). (A) The three models (i.e. the two baselines and the proposed STonKGs model) are trained and evaluated in a shared experimental setting. (B) For each text evidence and triple pair, the two baseline models exclusively use a single modality, whereas STonKGs leverages both

preprocessed version of the INDRA KG consisted of 174534 nodes and 13609994 triples. Of these triples, 127149 were selected for each of the eight fine-tuning tasks since these are the only triples that contain labels and thus, are suitable for a classification task. These triples have been manually curated to indicate context-specific information (i.e. annotation class) or the relation type (see Supplementary Table S2). The 13482845 remaining non-annotated triples were used in the unlabeled pre-training procedure (see Supplementary Text S1).

\subsection{Models}

As shown in Figure 1, all three models were operating under the same experimental conditions (i.e. in the same transfer learning setting, evaluated on the same tasks), with the exception of their utilized modalities. In contrast to the NLP- and KG-baselines (i.e. text evidence and triple-based models), STonKGs jointly builds upon both modalities. The following Sections outline our proposed STonKGs model architecture as well as the three baseline models used as a benchmark. All three models shared the same twofold training procedure, consisting of a pre-training and a fine-tuning part. The architectural change in the fine-tuning procedure was equivalent across all three models and consisted of placing a classification head (i.e. neural network components that process the output of a model to generate class labels) on top of the pre-trained model.

\subsubsection{NLP-Baseline}

The NLP-baseline was built on the pre-trained BioBERT v.1.1 model (Lee et al., 2019), a Transformer-based LM trained for 1 million steps on chunks of 512 tokens from a 4.5 billion token corpus stemming from PubMed abstracts (see Supplementary Table S3 for an overview on the hyperparameters of this model). To prepare the text evidence from INDRA statements for the NLP-baseline, the contiguous string of text was first split into single (sub)words (i.e. tokens), using the pre-trained tokenizer of BioBERT. The resulting token sequence was extended with special classification and separator tokens (i.e. [CLS] and [SEP]), and then padded or truncated accordingly to match the fixed input length of the LM (512 tokens, corresponding to a paragraph). Passing the sequence through BioBERT yielded token embedding vectors for a given text evidence, in which each of these embedding vectors is based on the weighted average of its surrounding tokens that is learnt by the attention mechanism of a pre-trained Transformer (Vaswani et al., 2017). This procedure ensures that each token embedding vector contains the context of its surrounding tokens.

To adapt BioBERT as a classifier for text evidence in a fine-tuning procedure, further model components, namely, pooling and a final linear layer with a softmax activation function, were added to enable sequence classification. In line with a commonly used aggregation technique derived from Devlin et al. (2019), our pooling procedure consists of using the special classifier (i.e. [CLS]) token embedding vector as a representation of the overall token embedding sequence for a given text evidence. This token embedding vector is used as an input for the final linear layer to generate class probabilities for the provided text evidence. Finally, we would like to note that in this transfer learning setting, we not only trained the parameters of the sequence classification components, but also fine-tuned all parameters of the entire model architecture, including the weights of the BioBERT model.

\subsubsection{KG-Baseline}

The inputs for the KG-baseline are high-dimensional node embeddings learnt by node2vec (Grover and Leskovec, 2016) using a walk length of 127 (all other hyperparameters are listed in Supplementary Table S3). Similar to the embeddings of word sequences produced by word2vec (Mikolov et al., 2013), node2vec generates embeddings for node sequences based on random walks. As a result, the embedding of a given node is formed based on the structure of its surrounding network neighborhood.

In concordance with the other two models, our KG-baseline relied on sequential inputs for each triple. Therefore, we designed a novel approach that generated a sequential representation for each triple while incorporating the embeddings generated by node $2 \mathrm{vec}$ (see Fig. 2). The general idea behind the sequential representation is to generate a sequence of embeddings $e\left(b_{i}, t_{i}\right)$ for the two nodes 
(1)-

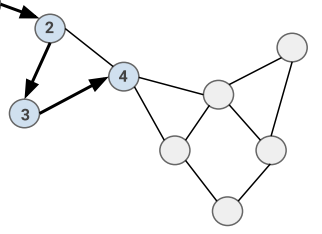

O

random walk-based embedding sequence
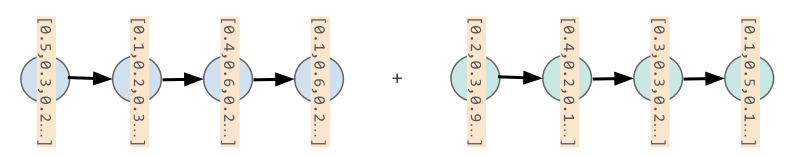

Fig. 2. Transforming $\mathrm{KG}$ embeddings into sequential inputs. For a given triple $\left(b_{i}, r_{i}, t_{i}\right)$, we generate the final random walk-based embedding representation $e\left(b_{i}, t_{i}\right)$ based on the following steps: (i) Obtain the random walks based on the pre-trained node2vec model: $h=\left(h_{i}, \ldots, h_{n}\right)$ and $t=\left(t_{i}, \ldots, t_{n}\right)$ for $h_{i}$ and $t_{i}$. (ii) Embed each node in those random walks, resulting in two random walk-based embedding sequences: $\overrightarrow{e_{h}}=\left(\overrightarrow{e_{h_{i}}}, \ldots, \overrightarrow{e_{b_{n}}}\right)$ and $\overrightarrow{e_{t}}=\left(\overrightarrow{e_{t_{i}}}, \ldots, \overrightarrow{e_{t_{n}}}\right)$. (iii) Generate the final embedding sequence $e\left(h_{i}, t_{i}\right)=\operatorname{concat}\left(\overrightarrow{e_{h}}, \overrightarrow{e_{t}}\right)=\left(\overrightarrow{e_{h_{i}}}, \ldots, \overrightarrow{e_{h_{n}}}, \overrightarrow{e_{t_{i}}}, \ldots, \overrightarrow{e_{t_{n}}}\right)$

$h_{i}, t_{i}$ in the $i$-th triple $\left(b_{i}, r_{i}, t_{i}\right)$. To do so, our proposed approach leveraged the sequence of random walks $h=\left(h_{i}, \ldots, h_{n}\right)$ and $t=$ $\left(t_{i}, \ldots, t_{n}\right)$ generated by node2 vec for $h_{i}$ and $t_{i}$, replacing each random walk by the embeddings $\overrightarrow{e_{b}}=\left(\overrightarrow{e_{b_{i}}}, \ldots, \overrightarrow{e_{b_{n}}}\right)$ and $\overrightarrow{e_{t}}=$ $\left(\overrightarrow{e_{t_{i}}}, \ldots, \overrightarrow{e_{t_{n}}}\right)$ learnt for each node in the walk. Subsequently, we acquired the embedding sequence of a given triple as the concatenation of the random walk-based embedding sequences of its two nodes $e\left(b_{i}, t_{i}\right)=\operatorname{concat}\left(\overrightarrow{e_{b}}, \overrightarrow{e_{t}}\right)=\left(\overrightarrow{e_{b_{i}}}, \ldots, \overrightarrow{e_{b_{n}}}, \overrightarrow{e_{t_{i}}}, \ldots, \overrightarrow{e_{t_{n}}}\right)$. This final random walk-based sequential representation, as opposed to other alternatives, ensured a fair comparison, since the other two models (i.e. NLP baseline and STonKGs) are also based on sequential inputs.

Similar to the NLP baseline outlined in Section 2.2.1, the embedding sequences for each triple are pooled, and passed through a linear layer with a softmax activation function to generate the final classification labels. Here, the pooling operation is defined as the dimension-wise maximum of the sequence embeddings, consequently mapping the sequence to a single vector. Since the KG-baseline uses static embeddings for the final classification task, the KG-baseline did not technically fit into the pre-training and finetuning paradigm used in NLP. However, for the sake of consistency, we will refer to the feature extraction based on transfer learning (i.e. embeddings from node2vec) used in the KG-baseline as pre-training and the final classification tasks as fine-tuning procedures as well.

To validate the effectiveness of our novel sequence representation approach, we conducted an additional study using TransE (Bordes et al., 2013) as an alternative KGEM. More specifically, this model architecture is generating an embedding sequence $\left(\overrightarrow{e_{b}}, \overrightarrow{e_{r}}, \overrightarrow{e_{t}}\right)$ by directly concatenating the embeddings of the head, relation and tail entities learnt by TransE (using the same embedding dimension as the original KG-baseline). Analogous to the sequence representations derived from node $2 \mathrm{vec}$, the TransE-based sequence is pooled using the dimension-wise maximum, and passed to the same classification architecture again.

\subsubsection{STonKGs}

Similar to BERT, STonKGs consists of multiple stacked Transformer layers with the attention mechanism forming the core of the overall model architecture. However, in contrast to the standard attention mechanism applied on text tokens, STonKGs uses a joint Transformer on a concatenation of text tokens and KG nodes, as illustrated in Figure 3. In accordance with the terminology introduced by Kamath et al. (2021) for their joint Transformer (on image and text data), this Transformer is hereafter referred to as a cross

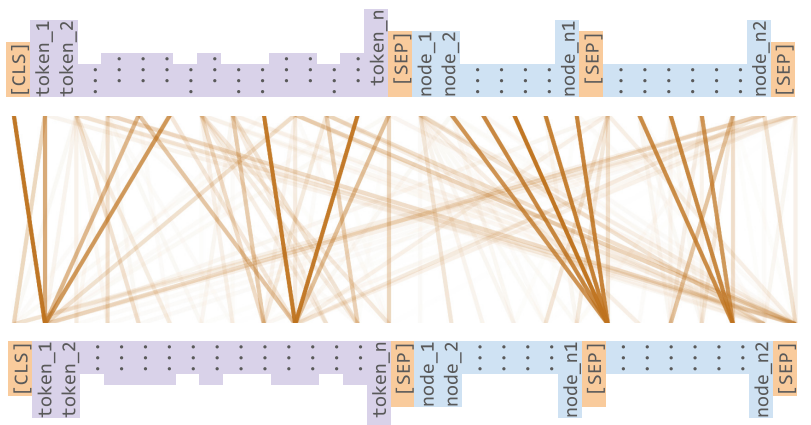

Fig. 3. Cross-modal attention between text data (token sequences) and KG data (triple sequences). The input is a concatenation of a token and a triple sequence. Each element in the initial input sequence consists of its respective BioBERT embedding. The resulting hidden states are processed by two different heads for text tokens and KG nodes, respectively. While the MLM head is returning probabilities for each token of the NLP-backbone, the MEM head is converting the hidden states onto probabilities for each node of the KG-backbone

encoder. The rationale behind using a cross encoder over other information fusion techniques was that it allows for learning implicit alignments between text tokens and KG nodes without requiring any entity linking step between the two modalities. More specifical$1 y$, the interdependencies in the combined input sequence are represented by attention weights, shown by the links between the inputs in Figure 3. These weights are learnable parameters used to calculate weighted average representation of a given entity (i.e. text token or node of the input sequence) based on the embedding vectors of its surrounding entities from both modalities. As a result, the calculated representation of each entity contains contextual information from the $\mathrm{KG}$ and text input.

To construct the cross encoder of STonKGs, we used the same hyperparameters as the BERT $\mathrm{BASE}_{\mathrm{B}}$ model (see Devlin et al., 2019), such as the maximum sequence length (512 tokens), hidden state dimension, number of Transformer layers and attention heads. We used embeddings of the combined text and KG input sequences as the input to STonKGs, based on the text-triple pairs extracted from the INDRA statements. The overall input sequence length was split into half, to comprise 256 text tokens and 256 $\mathrm{KG}$ nodes (including special tokens). The initial embedding sequences of the text-triple pairs were generated with BioBERT and node2vec for text and triples, respectively, which we will refer to as the NLP- and KG-backbone in the following (based on the steps outlined in Sections 2.2.1 and 2.2.2). However, instead of simply concatenating the random walk-based embedding sequences of the two nodes of a triple, we further added a [SEP] token between and after the two random walk sequences $\left(\overrightarrow{e_{h_{i}}}, \ldots, \overrightarrow{e_{h_{n}}},[S E P], \overrightarrow{e_{t_{0}}}, \ldots, \overrightarrow{e_{t_{n}}},[S E P]\right)$, as shown in Figure 3. The use of the special separator token intends to structurally differentiate between text and KG data in the input sequence, similar to the distinction of two input sentences in the original BERT model. Moreover, we masked some of the input using the embedding vector of the special [MASK] token from the NLP-backbone (the masking strategy is explained in detail below). In addition, we used positional and segment embeddings to further distinguish text and KG nodes of the combined input sequence in our cross encoder. Given the described inputs of STonKGs, the model has three different training objectives during pre-training, which are jointly used to learn the parameters of the cross encoder:

1. Masked Language Modeling (MLM): For the first 256 text tokens, we used the same MLM task and followed the same masking procedure used in the pre-training process of BERT. 
The goal of this task is to correctly predict the masked tokens based on a so-called MLM head. This head consists of a linear layer followed by the softmax function, which maps the final hidden states of the cross encoder to probabilities for each token in the vocabulary of the NLP-backbone.

2. Masked Entity Modeling (MEM): Inspired by the original MLM task, we built a counterpart for predicting masked nodes for the latter half of the combined input sequence (i.e. the KG input), again using the same masking strategy as in BERT. In this case, the goal is to correctly predict masked nodes in the random walk-based embedding sequences. Analogous to the MLM head, our custom MEM head consists of a linear layer followed by a softmax function. However, unlike the MLM head, the MEM head maps the hidden states to probabilities for each node occurring in the KG of the KG-backbone (as well as the [SEP] token, to remain consistent with the added [SEP] tokens) (see Fig. 3).

3. Next 'Sentence' Prediction (NSP): Similar to the original NSP task, we designed an equivalent training objective that aims to correctly predict whether a text and triple belong to each other, or whether they are randomly chosen from distinct INDRA statements. In accordance with Devlin et al. (2019), we also used the final hidden state of the [CLS] token for this binary prediction task. However, to preserve as much of the original training data as possible, we decided to augment the training data (rather than replace entries in it) with negative samples. In our case, we used $25 \%$ of the original pre-training dataset size, which is significantly smaller than the $50 \%$ used in BERT.

As a result, the pre-training objective of STonKGs consists of minimizing the total loss, more specifically, the sum of the losses across all three training objectives: $\mathcal{L}_{\text {total }}=\mathcal{L}_{M L M}+\mathcal{L}_{M E M}+\mathcal{L}_{N S P}$. All relevant hyperparameters used for the pre-training process of STonKGs (e.g. batch size and learning rate) are listed in Supplementary Table S3.

To evaluate STonKGs on each of the eight fine-tuning tasks (explained in the next section), we followed the same procedure that is outlined in Section 2.2.1 (NLP-baseline). Consequently, we used a classification head on top of the pre-trained STonKGs architecture, consisting of a pooling step, a linear layer and a softmax activation function to generate class probabilities for a given text-triple pair. Similar to the NLP-baseline, we also utilized the [CLS] token for pooling, and tuned all parameters of the entire STonKGs model architecture in our fine-tuning tasks.

\subsection{Evaluation}

In line with other Transformer-based transfer learning approaches, we used the majority of the INDRA text-triple pairs, predominantly unannotated triples, for pre-training (see Section 2.1), and the remaining annotated text-triple pairs (approximately $1.63 \%$ ) were used for the fine-tuning datasets. We evaluated the models on a benchmark consisting of eight fine-tuning tasks, namely, two relation-type classification tasks, four context annotation tasks and two correct/incorrect tasks (tasks 1-2, 3-6, 7-8 in Table 1, respectively). The relation-type tasks consist of two binary classifications in which each model either predicts the polarity (i.e. increase or decrease) or the type of interaction (i.e. direct or indirect interaction) of a given triple. Due to the direct encoding of the relation type, using a TransE-based approach on these two tasks would lead to an unfair advantage. Hence, we left out the relation-type tasks in the evaluation of TransE-based variants. The four context annotation tasks aim to predict the class (i.e. the context) of given text-triple pairs in a variety of biomedical settings: (i) cell line, (ii) disease, (iii) cellular location and (iv) species. All of these cases represent multiclass classification tasks using between three and ten classes depending on the most common occurrences of classes in each of the contexts. Finally, the two correct/incorrect tasks consist of a binary classification task where the model determines whether the text-triple pair is correct or incorrect, and a multiclass task where the model not only determines whether it is correct or incorrect but also which type of error it is. The sample sizes of the task-specific fine-tuning datasets ranged from 3760 to 78979 text-triple pairs, depending on the availability of triple annotations. An overview on the tasks as well as their respective summary statistics can be found in Supplementary Table S2. The distribution of classes of the fine-tuning tasks can be found in Supplementary Figure S2.

The performance of the models was evaluated on all eight classification tasks via a fivefold cross-validation procedure using weighted F1-scores (i.e. averages of the class-specific F1-scores weighted by number of true instances per class). To train and evaluate all three models on the same cross-validation splits, we created the splits deterministically (justifications for the model evaluation choices are given in Supplementary Text S2). All models were finetuned for five epochs on the training data using a batch size of 16 and the AdamW (Loshchilov and Hutter, 2019) optimizer with a linearly decreasing learning rate initially set to $5 * 10^{-5}$.

In addition to the proposed baselines, we run three ablation studies or variants of the STonKGs model to analyze the effect of certain model design choices on the fine-tuning tasks:

1. Fewer training steps: We created two versions of the STonKGs model, STonKGs $\mathrm{s}_{150 \mathrm{k}}$ and STonKGs $300 \mathrm{k}$, which were pre-trained for 150000 and 300000 steps (i.e. updates of the weights), respectively. More specifically, this was achieved through model checkpointing (i.e. STonKGs $s_{150 \mathrm{k}}$ is an interim checkpoint of STonKGs ${ }_{300 k}$ ). In doing so, we were able to observe the effect of reducing the number of training steps on the model performance in the fine-tuning procedures.

2. No NSP objective: Since the effectiveness of the NSP task for pre-training has been questioned (see Liu et al., 2019), we decided to design a variant of STonKGs $150 \mathrm{k}$ (termed

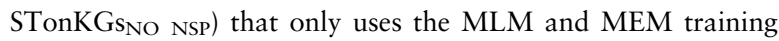
objectives. In result, this ablation measures whether the learned distinction between associated and randomly coupled text-triple pairs has an effect on fine-tuning task performances.

3. Different sequential representation of KG data: Similar to the second KG-baseline introduced in Section 2.2.2, we replaced the random walk-based embedding sequence in the overall input with a TransE-based representation of a triple in each text-triple pair. As a result, STonKGs TransE $_{\text {uses a direct con- }}$ catenation of head, relation and tail embeddings learnt by TransE (Supplementary Fig. S3). With this, we intended to analyze the effect of our novel sequence representation approach on the performance of STonKGs. Since both the TransE-based KG-baseline as well as STonKGs $s_{\text {TransE }}$ are directly encoding the relation type $r$, using the relation-type tasks (task 1-2) for evaluation would lead to an unfair advantage. Therefore, these two models were not evaluated on the polarity and interaction type tasks.

\subsection{Implementation details}

Both the NLP-baseline as well as STonKGs are implemented using the HuggingFace transformers library (v.4.6.1). More specifically, the NLP-baseline was initialized using the dmis-lab/biobert-v1.1 BioBERT model available at the HuggingFace model hub. For STonKGs, we leveraged the BertForPreTraining class as a basis, and modified its prediction heads and forward pass function. The STonKGs model was pre-trained on 4x NVIDIA A100 40GB Tensor Core GPUs. The pre-training procedure took $284.18 \mathrm{~h}$ ( 11.84 days) and $568.35 \mathrm{~h}$ (23.68 days) for STonKGs ${ }_{150 \mathrm{k}}$ and STonKGs ${ }_{300 \mathrm{k}}$, respectively. Finally, to set up the KG-baseline, we used the nodevectors library (v.0.1.23) for learning the random walk-based embedding sequences, and built a PyTorch Lightning (v.1.2.3) model on top. We trained our random walk-based embedding 
Table 1. Overview on the fine-tuning classification tasks

\begin{tabular}{|c|c|c|c|c|}
\hline Task & Description & Number of classes & Classes & Example \\
\hline (1) Polarity & $\begin{array}{l}\text { Directionality effect of the } \\
\text { source node on the target } \\
\text { node }\end{array}$ & Binary & Increase and decrease & $\begin{array}{l}\text { 'HSP70 }[\ldots] \text { increases ENPP1 } \\
\text { transcript and protein levels' } \\
\text { (PMID : 19083193) }\end{array}$ \\
\hline (2) Interaction type & $\begin{array}{l}\text { Whether it is known to be a } \\
\text { physical interaction between } \\
\text { the source and the target } \\
\text { node }\end{array}$ & Binary & Direct and indirect interaction & $\begin{array}{l}\text { 'SHP repressed [...] transcrip- } \\
\text { tion of PEPCK through dir- } \\
\text { ect interaction with C/ } \\
\text { EBPalpha protein' (PMID : } \\
\text { 17094771) }\end{array}$ \\
\hline (3) Cell line & $\begin{array}{l}\text { Cell line in which the given re- } \\
\text { lation has been described }\end{array}$ & 10 & $\begin{array}{l}\text { HEK293, DMS114, HeLa, } \\
\text { NIH-3T3, HepG2, MCF7, } \\
\text { COS-1, THP-1, LNCAP and } \\
\text { U-937 }\end{array}$ & $\begin{array}{l}\text { 'We show that upon stimula- } \\
\text { tion of HeLa cells by } \\
\text { CXCL12, CXCR4 becomes } \\
\text { tyrosine phosphorylated' } \\
\text { (PMID : 15819887) }\end{array}$ \\
\hline (4) Disease & $\begin{array}{l}\text { Disease context in which the } \\
\text { particular relation occurs }\end{array}$ & 10 & $\begin{array}{l}\text { Neuroblastoma, breast cancer, } \\
\text { lung cancer, atherosclerosis, } \\
\text { multiple myeloma, leuke- } \\
\text { mia, melanoma, osteosar- } \\
\text { coma, lung non-small cell } \\
\text { carcinoma }\end{array}$ & $\begin{array}{l}\text { '[ . . . nicotine }[\ldots] \text { activates } \\
\text { the MAPK signaling path- } \\
\text { way in lung cancer' (PMID : } \\
14729617 \text { ) }\end{array}$ \\
\hline (5) Location & $\begin{array}{l}\text { Cellular location in which the } \\
\text { particular relation occurs }\end{array}$ & 5 & $\begin{array}{l}\text { Cell nucleus, extracellular } \\
\text { space, cell membrane, cyto- } \\
\text { plasm and extracellular } \\
\text { matrix }\end{array}$ & $\begin{array}{l}\text { 'The activated MSK1 translo- } \\
\text { cates to the nucleus and acti- } \\
\text { vates CREB [...].' (PMID : } \\
\text { 9687510) }\end{array}$ \\
\hline (6) Species & $\begin{array}{l}\text { Species in which the particular } \\
\text { relation has been described }\end{array}$ & 3 & Human, mouse and rat & $\begin{array}{l}\text { 'Mutation of putative GRK } \\
\text { phosphorylation sites in the } \\
\text { cannabinoid receptor } 1 \\
\text { (CB1R) confers resistance to } \\
\text { cannabinoid tolerance and } \\
\text { hypersensitivity to cannabi- } \\
\text { noids in mice' (PMID : } \\
\text { 24719095) }\end{array}$ \\
\hline (7) Correct/Incorrect (Binary) & $\begin{array}{l}\text { Whether the extracted triple } \\
\text { correctly corresponds to the } \\
\text { text or not }\end{array}$ & Binary & Correct and incorrect & $\begin{array}{l}\text { Examples are available at } \\
\text { INDRA's curation guide- } \\
\text { lines (https://indra.readthe }\end{array}$ \\
\hline $\begin{array}{l}\text { 8) Correct/Incorrect } \\
\text { (Multiclass) }\end{array}$ & $\begin{array}{l}\text { Whether the extracted triple } \\
\text { correctly corresponds to the } \\
\text { text or not (including all } \\
\text { error types) }\end{array}$ & 8 & $\begin{array}{l}\text { Correct, no relation, wrong re- } \\
\text { lation, grounding, polarity, } \\
\text { act versus amt, entity boun- } \\
\text { daries, hypothesis }\end{array}$ & $\begin{array}{l}\text { docs.io/en/latest/tutorials/ } \\
\text { html_curation.html\#cura } \\
\text { tion-guidelines) }\end{array}$ \\
\hline
\end{tabular}

Note: While the two binary tasks (i.e. the polarity and interaction-type tasks) intend to evaluate the models' abilities to classify the relation type of the triple, the other four tasks deal with the classification of different types of contexts in which a given triple can appear in. Finally, the two tasks aim at predicting whether the triple has been correctly extracted from the text evidence.

sequences on a symmetric multiprocessing (SMP) node with four Intel Xeon Platinum 8160 processors and 1.5TB RAM.

\section{Results}

\subsection{Benchmarking}

To analyze the differences in performance across the models in our benchmark setting, it is important to understand the information that is exploited by each baseline model. While the KG-baseline aimed to represent topological node information, the NLP-baseline leveraged the unstructured textual information underlying the relations between the extracted named entities (e.g. 'Rosiglitazone directly increases Pdk4 transcriptional-levels in mice'). In our benchmark, six of the classification tasks consisted of predicting both types and context for each relation (e.g. a specific biological interaction is observed in a specific disease or species). Thus, the NLP-baseline seemed more suited for these tasks compared with the KG-baseline, since the information could explicitly be stated in the evidence itself. Indeed, this was confirmed by our results, where we observed a better performance of the NLP-baseline over the KGbaseline across all tasks. In addition, our proposed KG-baseline was limited by the use of static embeddings, as opposed to the transfer learning paradigm applied in both Transformer-based models (i.e. the NLP-baseline and STonKGs), which was based on fine-tuning the entire model architecture on given task-specific data. Below, we analyze the performances of the three presented models, as well as the ablated versions, across our proposed benchmark (Table 2).

First, we focus on the four more challenging classification tasks (i.e. those containing more than five classes), namely, tasks $3-5$ and 8 (see Table 2), where we observed that STonKGs considerably outperformed both baselines. Here, STonKGs achieved between 0.01 and 0.08 larger F1-scores compared with the NLP-baseline. Compared with the random walk-based KG-baseline, these differences were even larger resulting in F1-scores about $0.1-0.52$ higher for STonKGs. The TransE-based KG-baseline showed similar tendencies compared with the node-walk-based one, even though relation embeddings are included as an input. Specifically for the cell line and disease tasks (task 3 and 4), both KG-baseline variants failed to predict the correct entity class among the 10 possible classes, which was not the case for 
Table 2. Benchmark comparison of the baseline models and ablation variants of STonKGs on the chosen classification tasks

\begin{tabular}{|c|c|c|c|c|c|c|c|c|}
\hline \multirow[t]{2}{*}{ Model } & \multicolumn{2}{|c|}{ Relation type classification task } & \multicolumn{4}{|c|}{ Context annotation classification task } & \multicolumn{2}{|c|}{ Correct/incorrect classification task } \\
\hline & (1) Polarity & (2) Interaction type & (3) Cell line & (4) Disease & (5) Location & (6) Species & (7) Binary & (8) Multiclass \\
\hline NLP-Baseline (BioBERT) & 0.940 & 0.991 & 0.238 & 0.214 & 0.397 & 0.865 & 0.911 & 0.881 \\
\hline KG-Baseline (node2vec) & 0.448 & 0.945 & 0.020 & 0.030 & 0.295 & 0.670 & 0.708 & 0.446 \\
\hline KG-Baseline (TransE) & N/A & N/A & 0.046 & 0.081 & 0.320 & 0.534 & 0.485 & 0.195 \\
\hline STonKGs $_{300 k}$ & 0.930 & 0.995 & 0.252 & 0.248 & 0.405 & 0.860 & 0.977 & 0.964 \\
\hline STonKGs $_{150 \mathrm{k}}$ & 0.931 & 0.995 & 0.256 & 0.240 & 0.404 & 0.860 & 0.978 & 0.963 \\
\hline STonKGs $_{\text {NO NSP }}$ & 0.918 & 0.992 & 0.261 & 0.236 & 0.401 & 0.857 & 0.977 & 0.960 \\
\hline STonKGs $_{\text {TransE }}$ & N/A & N/A & 0.238 & 0.216 & 0.406 & 0.857 & 0.978 & 0.965 \\
\hline Absolute performance gain & -0.009 & +0.004 & +0.023 & +0.034 & +0.009 & -0.005 & +0.067 & +0.084 \\
\hline Relative performance gain & $-0.96 \%$ & $+0.40 \%$ & $+8.81 \%$ & $+15.89 \%$ & $+2.27 \%$ & $-0.58 \%$ & $+7.35 \%$ & $+9.53 \%$ \\
\hline
\end{tabular}

Note: Performance is measured as the average F1-score across the five cross-validation splits. For each classification task, the best model performance is highlighted in bold font. While the absolute performance gains are calculated based on the difference between the best STonKGs variant and the best baseline (i.e. the NLP baseline), the relative performance gains are obtained by dividing that difference by the F1-score of the best baseline and expressing the value as a percentage: $\frac{\left(S T O n K G s_{B E S T}-N L P\right)}{N L P} * 100$. If one of the STonKGs variants outperforms the baselines on a given task, the respective absolute and relative differences are highlighted in green, otherwise, they are colored in red.

task 5 and 8 , which both contain a lower number of classes. This suggests that it was particularly challenging for the KG-baselines to perform well across an increasing number of classes. On the other hand, when looking at the remaining four classification tasks containing only two or three classes (i.e. task 1-2 and 6-7), we observed that both the NLP-baseline as well as STonKGs resulted in higher F1scores than the KG-baselines. However, while STonKGs clearly outperformed the NLP-baseline with a difference of 0.067 on task 7 , there were only minimal differences between the two models across tasks 1-2 and 6. While the NLP-baseline led to a 0.009 and 0.005 improvement on the polarity and species tasks, STonKGs achieved a $0.004 \mathrm{~F} 1$-score improvement on the interaction task.

Interestingly, the random walk-based KG-baseline was only slightly worse than the other two models on the interaction type and species classification tasks. Moreover, the TransE-based KG-baseline approach resulted in an F1-score of 0.534 on the species task. In addition, on the polarity task, the performance of the random walkbased KG-baseline was similar to a random classifier. The relative increase in performance of the random walk-based KG-baseline on the interaction-type task compared with the polarity task can be attributed to imbalanced associations between nodes and class labels (e.g. a given node might be exclusively present in indirect interactions) (see Supplementary Fig. S4). Moreover, in the case of species classification, the relatively high performance of the KG-baselines, specifically of the random-walk-based one, was not surprising as the nodes in the INDRA KG can indirectly encode species information. For instance, protein nodes corresponding to the same ortholog gene were represented by species-specific identifiers [e.g. HGNC: PRKCG (human) and UP: P63319 (rat)]

When comparing STonKGs $\mathrm{S}_{300 \mathrm{k}}$ and STonKGs $\mathrm{S}_{150 \mathrm{k}}$, there was no significant difference in model performance. This was not surprising given the already low loss exhibited by STonKGs150k and the minor further reduction of the loss in STonKGs $300 \mathrm{k}$ (Supplementary

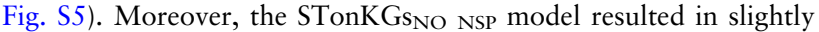
lower performances than the STonKGs $\mathrm{s}_{150 \mathrm{k}}$ model on almost all the evaluation tasks (apart from task 3), thus, suggesting that the NSP training objective was potentially beneficial for the overall pretraining procedure. In contrast, $\mathrm{STonKGs} \mathrm{TransE}_{\mathrm{T}}$ led to the same or improved performances compared with the other STonKGs variants on task $5-8$, hence it can be seen as a valid alternative approach. However, on task 3 and 4, STonKGs $s_{\text {TransE }}$ performed considerably worse, proving the effectiveness of the random-walk-based sequence representation of a triple in more complex tasks with a higher number of classes.

\subsection{STonKGs and applications}

The source code and trained models are, respectively, available at https://github.com/stonkgs/stonkgs and https://github.com/stonkgs/ results. The documentation is available at https://stonkgs.readthe docs.io/. The pre-trained STonKGs model can be downloaded from the HuggingFace model hub (https://huggingface.co/stonkgs/ stonkgs-150k).

To demonstrate the generalizability of the pre-trained STonKGs model (discussed in detail in Supplementary Text S3), we fine-tuned it on INDRA-independent text-triple pairs specific to two neurodegenerative indication areas (i.e. Alzheimer's disease, Parkinson's disease) (Domingo-Fernández et al., 2017) (presented in Supplementary Text S4). Furthermore, the fine-tuned STonKGs models, which are also released, can also be used to automatically annotate text-triple pairs with respect to the defined classes for each fine-tuning task (e.g. human, mouse and rat for the species context annotation task); thus, facilitating automatic annotations of biomedical KGs in a variety of contexts.

\section{Discussion}

In this work, we introduced STonKGs, a multimodal Transformer trained on millions of text-triple pairs from biomedical literature assembled by INDRA. STonKGs combines text and KG embeddings using a novel approach based on exploiting random walks learnt by node $2 \mathrm{vec}$ to generate sequential input data. We demonstrate the utility of our approach in a benchmark consisting of eight fine-tuning tasks. Here, STonKGs outperformed two baseline models, which were trained solely on either text or KG data, on the majority of the benchmark tasks. Each of the eight fine-tuning tasks represents a different classification problem with a specific biological use-case, hence confirming the generalizability of our proposed transfer learning approach. In addition to the benchmark, we conducted further ablation studies to measure the influence of the number of training steps and the NSP training objective on the overall performance of STonKGs. Finally, the source code and the pre-trained model are available at https://github.com/ stonkgs, enabling to leverage both the pre-trained STonKGs model as well as the overall model architecture for a variety of additional ML-based tasks that use text and KG data.

There exist some limitations to our proposed STonKGs model. First, while we have trained STonKGs on a novel and comprehensive KG that has not been utilized by any other Transformerbased model before, the INDRA KG is comparatively smaller than other large-scale available non-biomedical KGs such as Wikidata (Vrandečić and Krötzsch, 2014) and DBpedia (Bizer et al., 2009). This is mainly caused by the challenging tasks of recognizing biological entities and extracting their relations, given the ambiguity and complexity of biomedical jargon. Furthermore, INDRA aims at high precision and the used extraction process focuses on high quality rather than completeness. This impacted 
the text-triple pairs present in the fine-tuning datasets (i.e. some of them contain only several thousand text-triple pairs). Second, one characteristic property of the INDRA KG is that its textual evidence have been extracted on sentence level, consequently they are shorter in length compared with text sequences used in other Transformer-based LMs (e.g. Devlin et al., 2019; Zaheer et al., 2020). Given the complexity of biological scientific literature, the contextual representations learned by STonKGs could benefit from longer sequences (i.e. the context of a given triple is often mentioned in the surrounding sentences). Moreover, for some tasks, the small difference in performance between the NLPbaseline and STonKGs suggests that the textual evidence alone can be sufficient to tackle certain use cases. Third, while we have generated the node embeddings based on node2vec or TransE, other more sophisticated models such as Graph Convolutional Networks (GCNs) and Graph Attention Networks (GATs) (Ji et al., 2021) could be used. Moreover, the use of such models could overcome the lack of relation embeddings in node2 vec as well as the shortcomings of the purely translation-based TransE approach. However, this is practically infeasible due to the computational complexity required given the size of our KG. Furthermore, there are two requirements fulfilled by node2vec, but not by other models: (i) node2vec scales well for large-scale KGs, and (ii) sequential input is implicitly generated using random walks for each node. Another limitation is the absence of an optimization procedure for hyperparameters such as the batch size or the learning rate of STonKGs due to the run time implications (i.e. pre-training required several weeks, and running all benchmark tasks for STonKGs took more than a day). However, we demonstrated the effectiveness of STonKGs using the standard hyperparameters from the original BERT model. Finally, there are at least two reasons why we could not include other KG-extended Transformers (i.e. Fei et al., 2020; He et al., 2020; Zhang et al., 2019) in our benchmark setting: (i) these models require entity linking between text and KG nodes (see Introduction), and (ii) our benchmark is specifically designed to evaluate the performance of the models in classifying context and relation type information, which is not covered in benchmarks of other approaches.

Although we have demonstrated a proof-of-concept of our methodology across a variety of classification tasks, we would like to mention possible future improvements of STonKGs. First, the STonKGs pre-training procedure could potentially benefit from an even larger corpus of text-triple pairs. Due to our proposed transfer learning setting, additional corpora of text-triple pairs can be flexibly fed into the model by continuing the pretraining procedure. Second, while we have proposed a novel method to generate contextualized graph embedding sequences based on random walks from node2vec, more powerful KGE models could be potentially adapted to generate sequential input embeddings as well. Third, to maximize information gain, textual descriptions of the KG nodes could be added to the model in a straightforward manner. In addition, further tasks with closer resemblance to the expected real-world use-cases (i.e. tasks with a larger number of classes, or a dedicated 'unknown' class) can be added to the benchmark to assess the potential of STonKGs for future application scenarios. Finally, an in-depth analysis of the attention weights between the text tokens and KG nodes used in STonKGs could reveal valuable insights about the interdependencies between the two modalities.

\section{Acknowledgements}

The authors thank Sarah Mubeen for constructive criticism of the manuscript. Moreover, the authors thank Bruce Schultz for generating the INDRAindependent text-triple pairs. Finally, the authors thank André Gemünd for technical assistance running the experiments.

\section{Author contributions}

H.B. conceived and designed the study together with D.D.-F. H.B. implemented the methodology and conducted the experiments supervised by D.D.-
F. C.T.H., C.B., B.M.G. and J.B. assisted in defining the experiments. C.T.H., B.M.G. and J.B. provided and processed the data. M.H.-A., A.T.K., B.M.G., J.B. and D.D.-F. acquired the funding. H.B., C.B. and D.D.-F. wrote the manuscript. C.T.H., B.M.G., J.B. and P.G.P. reviewed the manuscript.

\section{Funding}

This work was supported by the Fraunhofer Cluster of Excellence 'Cognitive Internet Technologies' and the Defense Advanced Research Projects Agency (DARPA) Automating Scientific Knowledge Extraction (ASKE) program under award HR00111990009.

Conflict of Interest: DDF received salary from Enveda Biosciences.

\section{Data Availability}

We make the source code and the Python package of STonKGs available at GitHub (https://github.com/stonkgs/stonkgs) and PyPI (https://pypi.org/pro ject/stonkgs/). The pre-trained STonKGs models and the task-specific classification models are respectively available at https://huggingface.co/stonkgs/ stonkgs-150k and https://zenodo.org/communities/stonkgs.

\section{References}

Baker,S. et al. (2016) Automatic semantic classification of scientific literature according to the hallmarks of cancer. Bioinformatics, 32, 432-440.

Bizer,C. et al. (2009) DBpedia-a crystallization point for the Web of Data. J. Web Semant., 7, 154-165.

Bordes,A. et al. (2013) Translating embeddings for modeling multi-relational data. In: Proceedings of the 26th International Conference on Neural Information Processing Systems, Lake Tahoe, Nevada, United States. https://dl.acm.org/doi/10.5555/2999792.2999923. Accessed: 26 November 2020.

Dash,S. et al. (2019) Big data in healthcare: management, analysis and future prospects. J. Big Data, 6, 1-25.

Devlin,J. et al. (2019) BERT: pre-training of deep bidirectional transformers for language understanding. In: Proceedings of the 2019 Conference of the North American Chapter of the Association for Computational Linguistics: Human Language Technologies, Minneapolis, Minnesota, United States, Vol. 1, pp. 4171-4186.

Domingo-Fernández,D. et al. (2017) Multimodal mechanistic signatures for neurodegenerative diseases (NeuroMMSig): a web server for mechanism enrichment. Bioinformatics, 33, 3679-3681.

Doncheva,N.T. et al. (2021) Human pathways in animal models: possibilities and limitations. Nucleic Acids Res., 49, 1859-1871.

Federico,A. and Monti,S. (2021) Contextualized protein-protein interactions. Patterns, 2, 100153.

Fei,H. et al. (2020) Enriching contextualized language model from knowledge graph for biomedical information extraction. Brief Bioinformatics, 22, $1-14$.

Grover,A. and Leskovec,J. (2016) node2vec: scalable feature learning for networks. In: Proceedings of the 22nd ACM SIGKDD International Conference on Knowledge Discovery and Data Mining, San Francisco, California, United States, pp. 855-864.

Gyori,B.M. et al. (2017) From word models to executable models of signaling networks using automated assembly. Mol. Syst. Biol., 13, 954.

He,B. et al. (2020) BERT-MK: integrating graph contextualized knowledge into pre-trained language models. In: Findings of the Association for Computational Linguistics: EMNLP 2020, Online, pp. 2281-2290.

Ji,S. et al. (2021) A survey on knowledge graphs: representation, acquisition, and applications. IEEE Trans. Neural Netw. Learn. Syst., 2021, 1.

Kamath,A. et al. (2021). MDETR - Modulated Detection for End-to-End Multi-Modal Understanding. arXiv preprint arXiv:2104.12763.

Lee,J. et al. (2019) BioBERT: a pre-trained biomedical language representation model for biomedical text mining. Bioinformatics, 36, 1234-1240.

Li,J. et al. (2016) BioCreative V CDR task corpus: a resource for chemical disease relation extraction. Database, 2016, baw068.

Liu,Y. et al. (2019) Roberta: a robustly optimized bert pretraining approach. arXiv preprint arXiv:1907.11692.

Loshchilov,I. and Hutter,F. (2019) Decoupled weight decay regularization. arXiv preprint arXiv:1711.05101.

Mikolov,T. et al. (2013) Efficient estimation of word representations in vector space. arXiv preprint arXiv:1301.3781. 
Nadkarni,R. et al. (2021) Scientific language models for biomedical knowledge base completion: an empirical study. arXiv preprint arXiv: 2106.09700 .

Saqi,M. et al. (2019) Navigating the disease landscape: knowledge representations for contextualizing molecular signatures. Brief Bioinform., 20,609-623.

Stacey,R.G. et al. (2018) Context-specific interactions in literature-curated protein interaction databases. BMC Genomics, 19, 1-10.

Sun,T. et al. (2020) CoLAKE: Contextualized Language and Knowledge Embedding. arXiv preprint arXiv:2010.00309.

Tsatsaronis, G. et al. (2015) An overview of the BIOASQ large-scale biomedical semantic indexing and question answering competition. $B M C$ Bioinform., 16, 138.

Tsai,Y.-H.H. et al. (2019) Multimodal transformer for unaligned multimodal language sequences. In: Proceedings of the 57th Annual Meeting of the Association for Computational Linguistics, Florence, Italy, pp. 6558-6569.

Toutanova,K. et al. (2015) Representing text for joint embedding of text and knowledge bases. In: Proceedings of the 2015 Conference on Empirical Methods in Natural Language Processing, Lisbon, Portugal, pp. 1499-1509.
Vaswani,A. et al. (2017) Attention is all you need. In: Proceedings of the 31st International Conference on Neural Information Processing Systems, Long Beach, California, United States, pp. 6000-6010.

Vrandečić,D. and Krötzsch,M. (2014) Wikidata: a free collaborative knowl edgebase. Commun. ACM, 57, 78-85.

Wang,Z. et al. (2014) Knowledge graph and text jointly embedding. In: Proceedings of the 2014 Conference on Empirical Methods in Natural Language Processing, Doha, Qatar.

Wang,A. et al. (2018) GLUE: a multi-task benchmark and analysis platform for natural language understanding. In: Proceedings of the 2018 EMNLP Workshop BlackboxNLP: Analyzing and Interpreting Neural Networks for NLP, Brussels, Belgium, pp. 353-355.

Ying,C. et al. (2021). Do transformers really perform bad for graph representation? arXiv preprint arXiv:2106.05234.

Zhang,Z. et al. (2019) ERNIE: Enhanced Language Representation with Informative Entities. In: Proceedings of the 57th Annual Meeting of the Association for Computational Linguistics, Florence, Italy, pp. 1441-1451.

Zaheer,M. et al. (2020) Big bird: transformers for longer sequences. arXiv preprint arXiv:2007.14062. 